Original paper

\title{
Omega-3 fatty acid supplementation does not influence liver regeneration in rats after partial hepatectomy
}

\author{
Mohammad Akbari ${ }^{1}$, Suleyman Utku Celik², Akin Firat Kocaay ${ }^{1}$, Omer Arda Cetinkaya' ${ }^{1}$, Seher Demirer ${ }^{1}$ \\ 'Ankara University School of Medicine, Ankara, Turkey \\ ${ }^{2}$ Gulhane Training and Research Hospital, Ankara, Turkey
}

\begin{abstract}
Aim of the study: In the initiation of liver regeneration, multiple stimulatory and inhibitory factors participate. In this study, we aimed to evaluate the effects of omega- 3 fatty acids on liver regeneration after $30 \%$ partial hepatectomy in rats.

Material and methods: A total of 14 male Wistar Albino rats were included in this study. The animals were randomly allocated to two groups: the control group $(n=7)$ and the omega-3 group $(n=7)$. Rats in the control group were fed a standard rat chow and rats in the omega-3 group received $10 \mathrm{mg} / \mathrm{kg} /$ day omega-3 supplementation in addition to normal rat chow in the perioperative period. Rats were investigated seven days after $1 / 3$ partial hepatectomy by liver weight change and hepatocyte proliferation.

Results: The mean liver regeneration rate was found to be slightly higher $(p=0.061)$ in the omega-3 group compared the control group. In addition, no significant difference was observed regarding binuclear hepatocyte ratio in pericentral and periportal areas between the two groups. However, livers from rats given omega-3 supplementation have less inflammatory cellular infiltrate in the portal space than livers from the control group. Conclusions: Supplementation with omega-3 fatty acids showed no influence on the liver regeneration in rats undergoing $1 / 3$ partial hepatectomy.
\end{abstract}

Key words: liver regeneration, omega-3 fatty acids, rats.

\section{Address for correspondence}

Suleyman Utku Celik, MD, Clinic of General Surgery, Gulhane Training and Research Hospital, 06010 Ankara, Turkey, e-mail: s.utkucelik@hotmail.com

\section{Introduction}

Regeneration is a highly organized tissue regrowth process of the liver in response to tissue resection or injuries caused by hepatotoxic chemicals, drugs or infections [1]. The process is very complex and associated with cellular signaling cascades involving growth factors (such as transforming growth factor $\alpha$ and hepatocyte growth factor), cytokines (such as tumor necrosis factor $\alpha$ and interleukin 6), matrix remodeling, and harmonized stimulation and inhibition of growth-related signals [2, 3]. Moreover, liver regeneration is carried out by the proliferation of all existing mature cell types such as hepatocytes, biliary epithelial cells, vascular endothelial cells, stellate cells, Kupffer cells, macrophages, lymphocytes, and neuroendocrine cells $[2,4]$.
In physiological conditions, the mature hepatocytes are quiescent and do not proliferate; however, the process is triggered promptly after liver injury and different regenerative responses such as hyperplasia, hypertrophy, or reprogramming of a specific cell occur $[5,6]$.

Liver regeneration was first described in 1833 by the French pathologist Cruveilhier, who observed and explained hepatocyte proliferation in the liver of a dog which underwent partial hepatectomy $(\mathrm{PH})$ due to liver trauma [7]. In 1931, Higgins and Anderson most clearly demonstrated the regenerative capacity of the liver by establishing $\mathrm{PH}$ with resection of $2 / 3$ of the liver in rats $[7,8]$. Today, although two-thirds $(70 \%)$ $\mathrm{PH}$ has been used as a standard model for liver regeneration in experimental studies; resection of $1 / 3(30 \%)$, $1 / 2(50 \%)$, or $90 \%$ of the liver is also an acceptable 
procedure for studying regenerative organ growth [3, 9-11]. The removal of a part of the liver leads to a robust synchronous proliferative response involving the vast majority of the remaining hepatocytes in order to stimulate growth of the liver remnant $[1,9]$. Following hepatectomy, initial signals of liver regeneration can be observed within 5-10 minutes, lasting up to 5-7 days, and functional recovery of the regenerating liver can be monitored within 4 weeks after PH $[3,12]$. Hepatocytes are the first cells to enter the cell cycle and they produce mitogenic signals for other cell types [6]. The proliferation of the hepatocytes advances from the periportal to the pericentral region of the lobule, as a wave of mitosis $[6,13]$. Biliary epithelial cells, endothelial cells, stellate cells, and Kupffer cells undergo division a few days later than hepatocytes $[3,13]$. Normal liver weight is completely re-established within one week in rats and 8-15 days in humans following hepatectomy [14]. Lobular reorganization takes place slowly for several weeks, and eventually the liver histology becomes indistinguishable from the original [3].

Fatty acids, as essential nutrients, have many biological functions in health and disease, and omega-3 fatty acid $(\omega-3)$ supplementation is also involved in modifying the organic biochemical environment [15]. Furthermore, experimental studies have shown promising effects of omega- 3 fatty acids with regard to the prevention and reversal of hepatic steatosis, improvement in the regenerative capacity of the liver, and reducing ischemia-reperfusion injury following $\mathrm{PH}[16$, 17]. Omega- 3 fatty acids are also reported to alter the production of inflammatory mediators such as growth factors, chemokines, and matrix proteases and have anti-inflammatory effects [18].

Partial hepatectomy is the most commonly performed surgical procedure for the treatment of liver diseases such as hepatocellular carcinoma, cholangiocellular carcinoma, liver metastasis, liver trauma, and liver transplantation. Malnutrition is common in patients with the advanced liver diseases mentioned above. Therefore, perioperative nutritional support can significantly reduce the postoperative complications and mortality in patients with liver resection [19]. In this study, we aimed to evaluate the effects of omega-3 fatty acid administration on liver regeneration after $30 \%$ partial hepatectomy in rats.

\section{Material and methods}

\section{Ethics statement}

This study was approved by the Animal Experiments Ethics Committees of Ankara University School of Medicine (approval number: 2008-18-100) and the study was carried out in compliance with the Guide for the Care and Use of Laboratory Animals published by the US National Institutes of Health.

\section{Animals, experimental protocol, and study design}

The study was designed as a prospective, randomized, controlled animal trial with 1:1 allocation ratio and performed in the animal laboratory of Ankara University School of Medicine in Ankara, Turkey. A total of 14 male Wistar Albino rats weighing 275 to $325 \mathrm{~g}$ were used in this study. Rats were maintained in a room at a temperature of $22 \pm 2^{\circ} \mathrm{C}$, with a $12 \mathrm{~h} \mathrm{light-}$ dark cycle and relative humidity of $50 \%$ to $60 \%$ and with free access to water and standard chow.

The animals were randomly allocated to two groups ( $n=7$ in each group): the control group (only partial hepatectomy) and the omega-3 group (omega-3 fatty acid + partial hepatectomy). Rats in the control group were fed a standard rat chow ad libitum for 28 days. Rats in the omega-3 group received $10 \mathrm{mg} / \mathrm{kg} /$ day omega-3 supplementation (Marincap, Kocak Farma, Turkey) in addition to normal rat chow for 28 days. On the $21^{\text {st }}$ day of the study, all rats underwent $1 / 3$ partial hepatectomy (left lateral lobectomy). On the $28^{\text {th }}$ day, all rats in the two groups underwent total hepatectomy and were sacrificed afterward.

\section{Surgical technique}

The surgical procedures were performed between 9:00 and 11:00 am under heat lamps to maintain the body temperature within $35-36^{\circ} \mathrm{C}$ by the same surgeon with the same technique and under sterile conditions.

After $50 \mathrm{mg} / \mathrm{kg}$ intramuscular ketamine hydrochloride (Ketalar, Parke-Davis, Eczacibasi, Istanbul, Turkey) was given preoperatively for anesthesia, surgical interventions were performed in appropriate sterile conditions. Abdominal hair was trimmed and skin was cleaned with $10 \%$ povidone-iodine solution preoperatively in all rats. Laparotomy was performed with a $3 \mathrm{~cm}$ midline incision. After the liver was mobilized, the pedicles of the left lateral lobe were ligated with a suture and the liver tissue was transected. The abdomen closures were made with $4 / 0$ polypropylene sutures. Then, the resected liver tissue was weighed. In the morning of postoperative day 7 (on the $28^{\text {th }}$ day of the study), the animals were again anesthetized with the same method, and a laparotomy was performed through the previous incision. The remnant liver was removed, and the weight 
of the total remnant liver was noted. The resected liver was fixed in phosphate-buffered formalin for 24-48 h. Sacrifications were performed by injecting overdoses of ketamine intramuscularly.

\section{Liver weight and regeneration rate}

The preoperative estimated liver weight was calculated from the resected liver weight as $\mathrm{ELW}_{\text {pre-op }}=\mathrm{LW}_{\text {resect }} / 0.3$. The change in liver weight was estimated as the liver regeneration rate $(\mathrm{RR})$ defined as $\mathrm{RR}=\left(\left(\mathrm{LW}_{\text {remn }} / \mathrm{BW}_{\text {euth }}\right)\right.$ / $\left.\left(\mathrm{ELW}_{\text {pre-op }} / \mathrm{BW}_{\text {pre-op }}\right)\right) \times 100 \%$ [20-22], where $\mathrm{ELW}_{\text {pre-op }}$ estimated liver weight; $\mathrm{LW}_{\text {resect }}$ - resected liver weight; $\mathrm{LW}_{\text {remn }}$ - remnant liver weight; $\mathrm{BW}_{\text {euth }}$ - body weight at euthanization; $\mathrm{BW}_{\text {pre-op }}$ - body weight preoperatively.

\section{Histological analysis}

After all surgical interventions, liver samples fixed in buffered $10 \%$ formaldehyde ( $\mathrm{pH} 7.4$ ) were embedded in paraffin, cut, and stained with hematoxylineosin (H-E), periodic acid-Schiff (PAS), and PAS with diastase (PAS-D) for routine histological examination, investigation of glycogen contents of the hepatocytes, and examination of Kupffer cells, respectively. Cross-sections were prepared from periportal and pericentral areas separately. All cross-sections were also examined for mononuclear $(\mathrm{Mn})$ and binuclear $(\mathrm{Bn})$ hepatocytes and assessed by microscopic observations and manual counting. Each analysis was conducted on a minimum of 500 cells per specimen for each animal. The binuclear hepatocyte to total hepatocyte ratio was calculated as $(\mathrm{Bn} / \mathrm{Mn}+\mathrm{Bn}) \times 100$.

\section{Statistical analysis}

Data were expressed as mean (standard deviation) for continuous variables and frequency (percentage) for categorical variables. The Shapiro-Wilk test was used to assess normality assumption for continuous variables. All numerical data were analyzed using Student's $t$-test. The Mann-Whitney $U$ test was used to evaluate differences between groups in the case of non-normally distributed continuous variables. Any $p$ value $<0.05$ was taken as significant. All statistical data were analyzed using SPSS version 16.0 for Windows (SPSS Inc., Chicago, IL) software.

\section{Results}

During the study, no animal died; all rats were healthy and behaved as expected with respect to food consumption and standard activity.
Table 1. Measures of the resected liver weights of rats in the two groups

\begin{tabular}{lcc}
\hline & Mean \pm SD (grams) & $p$ \\
\hline Control group & $6.82 \pm 1.57$ & 0.328 \\
\cline { 1 - 2 } Omega-3 group & $7.49 \pm 0.75$ & \\
\hline
\end{tabular}

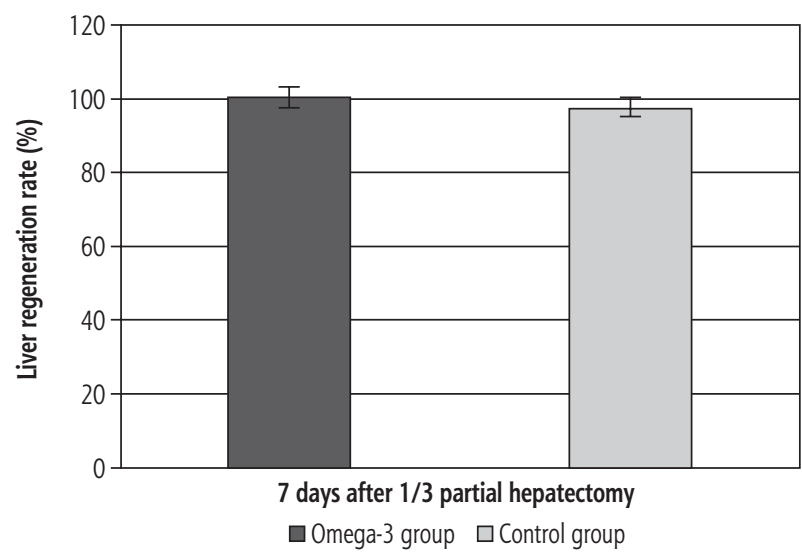

Fig. 1. Liver regeneration rate seven days after partial hepatectomy. Values are expressed as means \pm SD in log scale

Preoperative mean body weight of the rats was $300.3 \pm 14.6 \mathrm{~g}$ (275-325). Mean weight of the resected $1 / 3$ liver was $6.82 \pm 1.57 \mathrm{~g}$ in the control group and 7.49 $\pm 0.75 \mathrm{~g}$ in the omega- 3 group (Table 1 ). The mean liver regeneration rate at 7 days of $\mathrm{PH}$ was found to be slightly higher $(p=0.061)$ in the omega-3 group compared to the control group (100.5\% vs. 97.5\%) (Fig. 1).

Hematoxylin-eosin staining of remnant liver tissue in all rats revealed normal lobular architecture and hepatocyte morphology. A slight proliferation of the bile ducts was also noted in the portal areas in both groups (Fig. $2 \mathrm{~B}$ and $3 \mathrm{~B}$ ). One unanticipated finding was that livers from rats given omega-3 supplementation have less inflammatory cellular infiltrate in the portal space than livers from the control group (Fig. 2C and 3C).

Comparison of $\mathrm{Mn}$ and $\mathrm{Bn}$ hepatocyte count values and Bn-to-total hepatocyte ratio are listed in Table 2. $\mathrm{Bn}$ hepatocyte ratio was higher in the omega-3 group compared to the control group in pericentral and periportal areas. However, no significant difference was found between groups $(p=0.486$ and $p=0.586$, respectively).

PAS staining demonstrated that the livers of all rats had glycogen-rich hepatocytes around the central veins and glycogen-poor hepatocytes in the periportal region. However, dilatation of the sinusoidal space with inflammatory cell infiltration in the periportal region of the control group was significantly higher than that of the omega-3 group (Fig. 2D and 3D). In addition, PAS-D stain demonstrated an increase in the size of the Kupffer cells in rats fed with omega-3. 

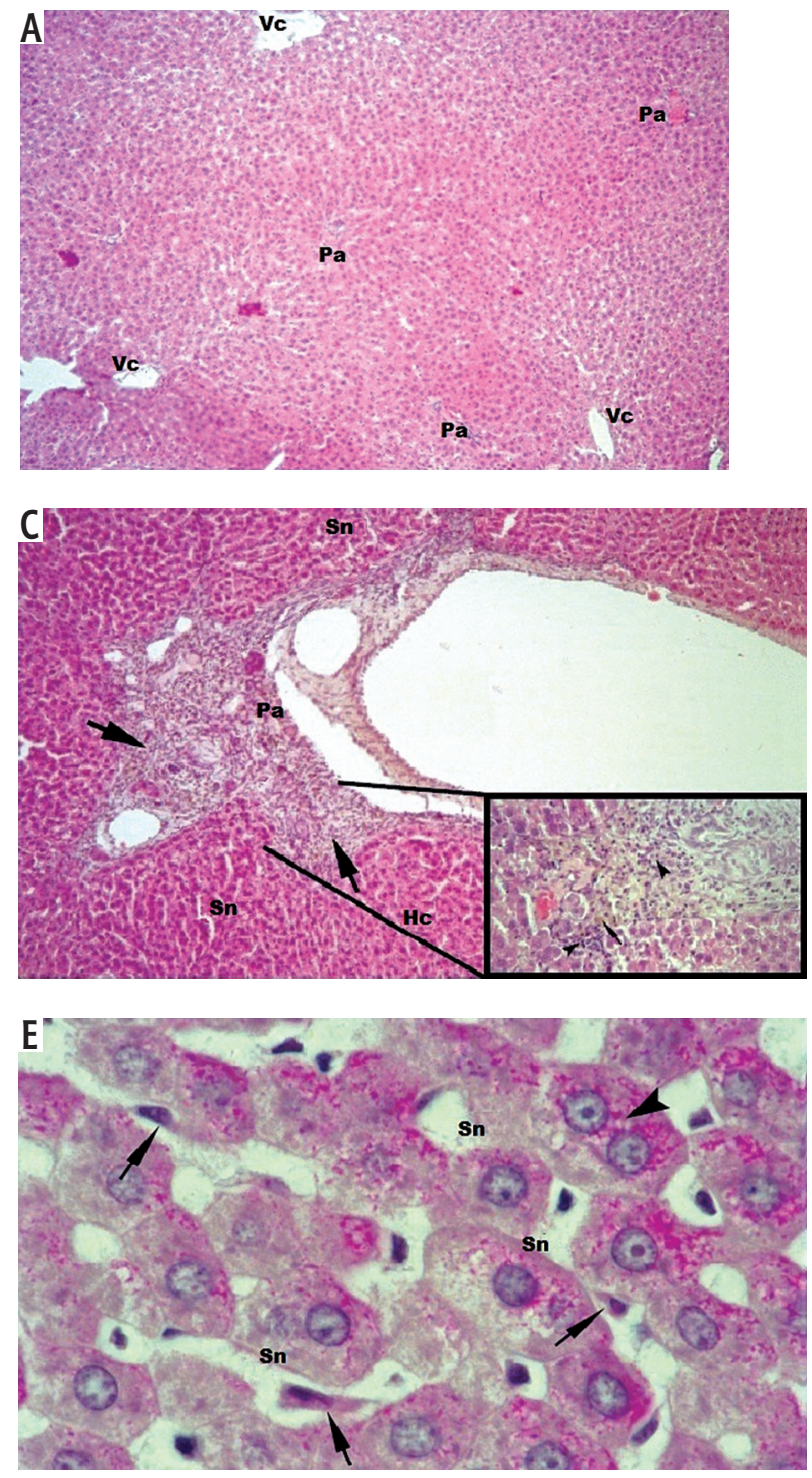

\section{Discussion}

Today, a liver resection, or $\mathrm{PH}$, has become the standard treatment for benign and malignant liver diseases, hepatic trauma, and also liver transplantation donors, and it is safely accomplished since the liver has the unique potential to regain its functional capacity in a matter of days or weeks $[2,23]$. In this context, it is of crucial importance to understand the complex process of liver regeneration. Thus, experimental models such as the $\mathrm{PH}$ are widely used in a relatively high number of homogeneous rats, and the regenerative process is assessable through the remnant liver [20].

In the initiation of liver regeneration, multiple stimulatory and inhibitory factors participate. Each factor plays a unique role in a signal cascade pattern. The best-understood mechanisms involved in liver cell proliferation and regeneration include orchestration
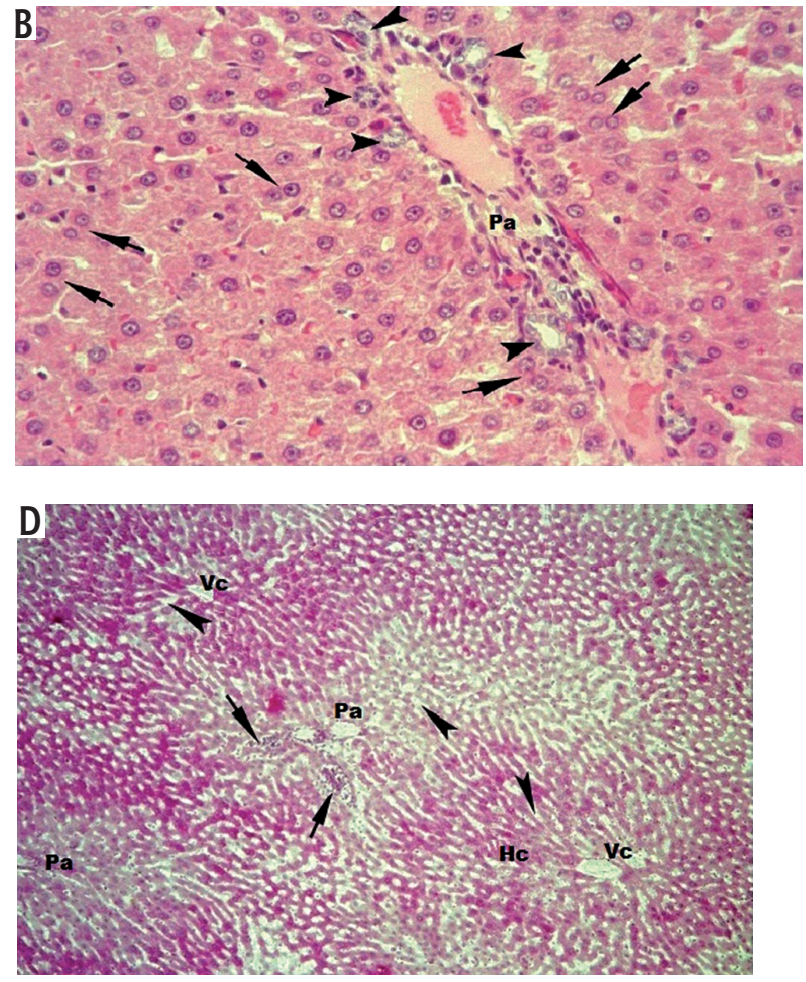

Fig. 2. Histological findings of livers taken from rats in control group. A) Normal lobular architecture and hepatocyte morphology with typical hepatic lobule, radial liver cell cord, and clear hepatic sinusoid (H-E; 10x). B) Slight proliferation of the bile ducts (arrowheads) and hepatocytes containing binuclear nucleus (arrows) in the portal area (H-E; 40x). C) Inflammatory cell infiltration in the portal area (arrows) (H-E; 10x). The inset shows increased hemosiderin-laden macrophage (arrow) and lymphocyte infiltration (arrowheads) (H-E; 40x). D) Glycogen-rich hepatocytes around central veins and glycogen-poor hepatocytes and dilated sinusoidal space with inflammatory cell infiltration in the periportal area (PAS; 10x). E) Kupffer cells (arrows) and hepatocytes containing binuclear nucleus (arrowhead) (PAS-D; 100x). Vc - vena centralis, $\mathrm{Pa}$ - portal area, $\mathrm{Sn}$ - sinusoid, $\mathrm{Hc}$ - hepatic cell cord

of a broad range of cellular factors such as hepatocyte growth factor (HGF), epidermal growth factor receptor (EGF), nuclear factor-kB (NF-kB), tumor necrosis factor (TNF), and interleukin 6. In addition, serotonin, norepinephrine, bile acids, leptin, and insulin are essential for hepatic regeneration $[1,11,13]$.

As briefly mentioned above, liver regeneration and its mechanism after $\mathrm{PH}$ in animals have been extensively studied for more than 90 years. In this period, several factors have been reported to promote liver regeneration such as prostaglandin E, vitamin E, branched-chain amino acid supplementation, oral supplements of L-arginine and L-glutamine, fish oil, and zinc supplementation [24-28].

Recent experimental studies have indicated that omega-3 polyunsaturated fatty acids (PUFA) have anti-inflammatory effects, reduce hepatic steatosis, improve the regenerative capacity of the liver, and in- 

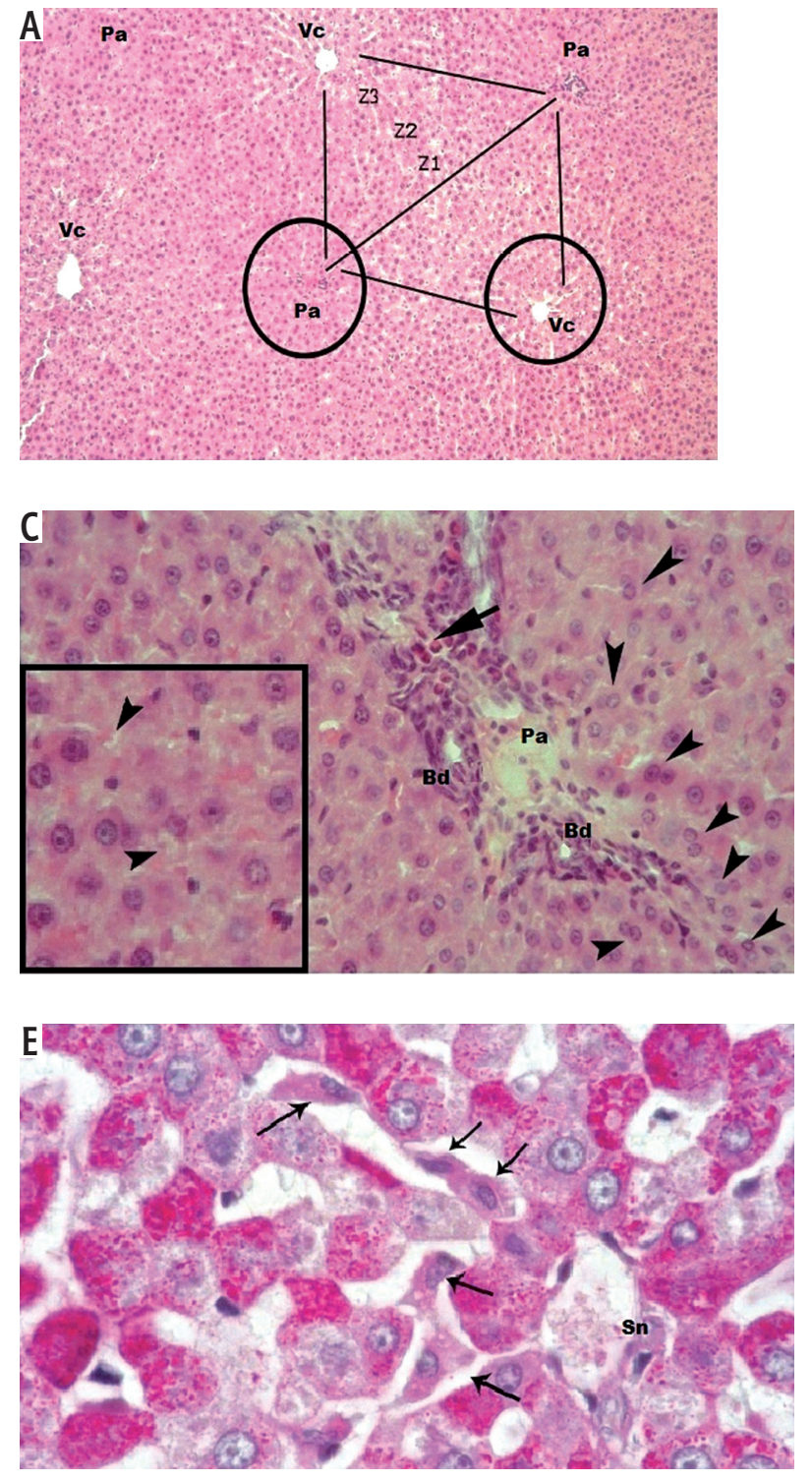

crease the tolerance toward ischemia-reperfusion injury $[16,17]$. Moreover, it has been shown that omega-3 PUFA plays a crucial role in gastrointestinal surgery; it protects hepatocytes through inhibiting liver cell peroxidation via anti-oxidation and anti-inflammatory mechanisms of action after $\mathrm{PH}$ [29]. Nevertheless, there has been only limited evidence about the effects of omega-3 PUFA on the regeneration of the liver. This study focused on the role of omega- 3 fatty acids in liver regeneration after one-third partial hepatectomy.

It should be considered that, in this study, $\mathrm{PH}$ was used only as an inducer of cell proliferation. Many investigators have used $2 / 3 \mathrm{PH}$ as an inducing factor for liver regeneration in rat models $[1,5,10,20,21,23]$. This method is relatively easy to carry out and it results in high proliferation rates of hepatocytes $[1,10]$. How-
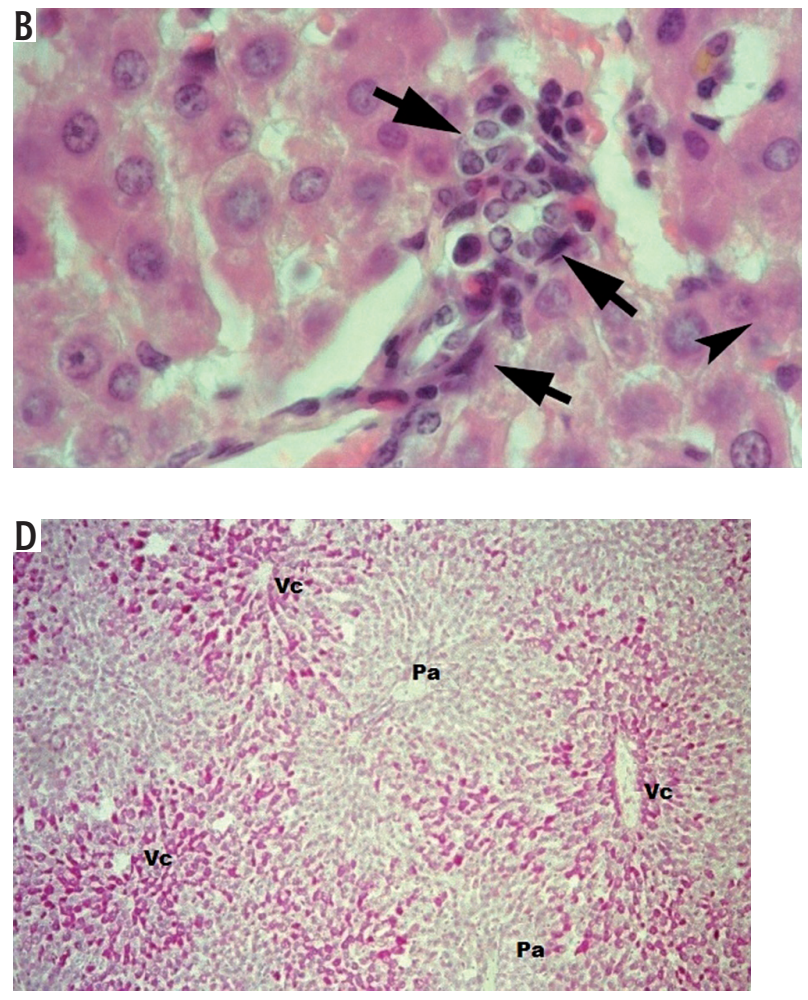

Fig. 3. Histological findings of livers taken from rats in omega-3 group. A) Normal lobular architecture and hepatocyte morphology (H-E; 10x). B) Bile duct proliferation (arrows) and binuclear hepatocytes (arrowhead) in the portal area (H-E; 100x). C) Binuclear hepatocytes (arrowheads), bile ducts, infiltration of eosinophil leukocyte in connective tissue (arrows), and nuclear pleomorphism ( $\mathrm{H}-\mathrm{E} ; 40 \mathrm{x})$. The inset shows multinuclear hepatocytes and sinusoids (arrowheads) (H-E; 100x). D) Glycogen-rich hepatocytes in the pericentral area and glycogen-poor hepatocytes in the periportal area (PAS; 10x). E) Kupffer cells located inside the sinusoids (arrows) (PAS-D; 100x). Pa - portal area, Vc - vena centralis, Z1 - zone 1 (periportal lobule), Z2 - zone 2 (midzonal region), Z3 - zone 3 (centrilobular area), Bd - bile duct, $\mathrm{Sn}$ - sinusoid

Table 2. Comparison of mononuclear and binuclear hepatocyte count values in pericentral and periportal areas of two groups

\begin{tabular}{lccc}
\hline Variable & $\begin{array}{c}\text { Control group, } \\
n=7\end{array}$ & $\begin{array}{c}\text { Omega-3 group, } \\
n=7\end{array}$ & $p$ \\
\hline Pericentral areas & & & \\
\hline $\mathrm{Mn}$ & $40.00 \pm 9.27$ & $45.29 \pm 7.63$ & 0.267 \\
\hline $\mathrm{Bn}$ & $3.86 \pm 1.34$ & $4.43 \pm 1.61$ & 0.486 \\
\hline Bn/Total & $8.8 \%$ & $8.9 \%$ & \\
\hline Periportal areas & & & \\
\hline $\mathrm{Mn}$ & $44.43 \pm 8.38$ & $46.29 \pm 8.71$ & 0.692 \\
\hline $\mathrm{Bn}$ & $4.43 \pm 1.61$ & $5.00 \pm 2.16$ & 0.586 \\
\hline $\mathrm{Bn} /$ Total & $9.1 \%$ & $9.7 \%$ & \\
\hline
\end{tabular}

$M n$ - mononuclear hepatocytes; $B n$ - binuclear hepatocytes 
ever, $2 / 3 \mathrm{PH}$ results in significantly greater systemic catabolic response, hypoglycemia, and accumulation of hepatic triglyceride along with more extensive proliferation of liver than $1 / 3 \mathrm{PH}$. Therefore, we preferred using one-third $\mathrm{PH}$ in this study, considering the disadvantages of two-thirds $\mathrm{PH}$, such as increased risk of severe hemodynamic deterioration, malnutrition, and over-induction of cell proliferation [30-32].

In the present study, two independent markers were used to assess proliferation: 1) Regeneration of the liver was expressed as the percentage of regenerated liver mass and was calculated as described previously by Kwon et al. [22]. 2) The number of binuclear hepatocytes and binuclear-to-total hepatocyte ratio, a traditional but still debated model, were considered as a sign of liver regeneration and compared between the two groups [10]. In contrast to earlier findings, however, this experiment did not reveal any significant difference between the omega-3 group and the control group in terms of the regeneration rate $(p=0.061)$. Furthermore, no significant difference was observed between groups with regard to hepatocyte counts. Overall, our results show that omega-3 PUFA supplementation could not influence the liver regeneration in rats after $1 / 3$ partial hepatectomy.

In recent years, there has been an increasing amount of literature describing promising effects of omega-3 fatty acids on liver regeneration following $\mathrm{PH}$, although contrasting results have been reported $[16,17,29]$. In a study which set out to examine the impact of the intravenous injection of omega-3 PUFA on liver regeneration, Qiu et al. [29] found that omega-3 PUFA could slow the progress of acute liver failure through an anti-inflammatory action and promote liver regeneration after $90 \%$ hepatectomy in rats. Similarly, Linecker et al. [17] found that two-thirds partial hepatectomized rats fed with omega-3 PUFA developed an increased tolerance toward ischemia-reperfusion injury, an improved regenerative capacity, and a trend toward lower mortality due to liver tumors. Moreover, in a study by Marsman et al. [16], it was revealed that omega-3 fatty acids effectively reverse experimental hepatic steatosis, resulting in improved liver functional and regenerative capacity.

To date, there have been only a few clinical studies assessing the impact of omega-3 PUFA on liver regeneration. One study by Gong et al. [19] examined omega-3 PUFA-based lipid emulsions for treatment of patients after $\mathrm{PH}$, and they found that these supplements are safe and effective in controlling inflammation, protecting liver function, and consequently reducing the risk of complications and the length of the hospital stay. In a randomized controlled study of parenteral nutrition support with omega- 3 fatty acids in patients who underwent liver transplantation for the diagnosis of end-stage liver disease or hepatic cellular carcinoma, Zhu et al. [33] reported that post-transplant nutritional support could significantly improve the injury of transplanted liver, decrease the infectious morbidities, and shorten the hospital stay. Similar results were also found by Ibrahim et al. [15].

In agreement with our results, Silva et al. [34] observed no significant effect of supplementation with omega-3 fatty acids in rats undergoing $60 \%$ hepatic resection on the outcome of liver regeneration. Similarly, Melo et al. [35] found that intraperitoneal infusion of omega-3 PUFA inhibited liver regeneration and induced reduction of hepatic reduced glutathione concentration, probably through inhibition of oxidative stress following $\mathrm{PH}$ in $70 \%$ partially hepatectomized rats.

Finally, a number of important limitations need further discussion and exploration. First, the liver regeneration was examined at only one time point (7 days after PH). Another limitation was that hepatocyte proliferation of the remnant liver was assessed only using light microscopy, without the use of immunochemistry. However, liver regeneration can be evaluated by several methods, such as the number of mitoses, components of deoxyribonucleic acid, synthesis rate of nuclear antigens, changes in serum protein levels, enzymatic determination of proliferation markers, serological tests, and flow cytometry. Moreover, the rat liver differs in size and anatomy, and its metabolic activity is much higher than that of human liver. Thus, the regenerative process of the liver in rats has almost been completed within 7 days following $\mathrm{PH}$, in contrast to humans. In addition, other uncertainties are due to the lack of information about the daily dose of omega-3 PUFA required to improve postoperative recovery and hepatocyte proliferation, duration of treatment, and basal omega- 3 status of the rats.

\section{Conclusions}

Based on our findings, together with previous observations, there is still conflicting and inconsistent evidence on the relationship between dietary intake of omega- 3 fatty acids and regeneration of the liver. However, this study did not show any significant improvement in the regeneration rate of the liver in rats fed with omega-3 PUFA supplementation after $1 / 3$ partial hepatectomy. Further experimental studies with larger samples are needed to demonstrate the effectiveness of omega-3 PUFA in liver regeneration, as well as to examine its mechanisms of action. 


\section{Acknowledgments}

The authors would like to thank Oya Evirgen for expert help in histological evaluation and Ferda Topal Celikkan for technical assistance.

\section{Disclosure}

Authors report no conflict of interest.

\section{References}

1. Fausto N, Campbell JS, Riehle KJ. Liver regeneration. Hepatology 2006; 43: 45-53.

2. Taki-Eldin A, Zhou L, Xie HY, Zheng SS. Liver regeneration after liver transplantation. Eur Surg Res 2012; 48: 139-153.

3. Michalopoulos GK. Liver regeneration. J Cell Physiol 2007; 213: 286-300.

4. Preziosi ME, Monga SP. Update on the mechanisms of liver regeneration. Semin Liver Dis 2017; 37: 141-151.

5. Martins PN, Theruvath TP, Neuhaus P. Rodent models of partial hepatectomies. Liver Int 2008; 28: 3-11.

6. Gilgenkrantz H, Collin de l'Hortet A. Understanding liver regeneration: from mechanisms to regenerative medicine. Am J Pathol 2018; 188: 1316-1327.

7. Power C, Rasko JE. Whither prometheus' liver? Greek myth and the science of regeneration. Ann Intern Med 2008; 149: 421-426.

8. Nevzorova YA, Tolba R, Trautwein C, et al. Partial hepatectomy in mice. Lab Anim 2015; 49: 81-88.

9. Riehle KJ, Riggle K, Campbell JS. The priming and progression theory of liver regeneration. In: Liver regeneration. Udayan A (ed.). Academic Press, Boston 2015; 50-63.

10. Miyaoka Y, Miyajima A. To divide or not to divide: revisiting liver regeneration. Cell Div 2013; 8: 8 .

11. Cao H, Yu J, Xu W, et al. Proteomic analysis of regenerating mouse liver following 50\% partial hepatectomy. Proteome Sci 2009; 7: 48.

12. Kocabayoglu P, Zhang DY, Kojima K, et al. Induction and contribution of $\beta$-PDGFR signaling by hepatic stellate cells to liver regeneration after partial hepatectomy in mice. Liver Int 2016. 36: $874-882$

13. Michalopoulos GK. Hepatostat: Liver regeneration and normal liver tissue maintenance. Hepatology 2017; 65: 1384-1392.

14. Tannuri AC, Tannuri U, Coelho MC, et al. Experimental models of hepatectomy and liver regeneration using newborn and weaning rats. Clinics 2007; 62: 757-762.

15. Ibrahim ES, Saleh SM, El Hoseeny M, et al. Effect of omega- 3 on hepatic regeneration in adult living donors undergoing hepatic resections for liver transplantation: A randomized controlled trial. J Crit Care 2016; 31: 157-162.

16. Marsman HA, de Graaf W, Heger M, et al. Hepatic regeneration and functional recovery following partial liver resection in an experimental model of hepatic steatosis treated with omega-3 fatty acids. Br J Surg 2013; 100: 674-683.

17. Linecker M, Limani P, Kambakamba P, et al. Omega-3 fatty acids protect fatty and lean mouse livers after major hepatectomy. Ann Surg 2017; 266: 324-332.

18. El-Magd NFA, El-Karef A, El-Shishtawy MM, et al. Hepatoprotective effects of glycyrrhizin and omega- 3 fatty acids on Nuclear Factor-kappa B pathway in thioacetamide-induced fibrosis in rats. Egypt J of Basic Appl Sci 2015; 2: 65-74.
19. Gong Y, Liu Z, Liao Y, et al. Effectiveness of $\omega$-3 polyunsaturated fatty acids based lipid emulsions for treatment of patients after hepatectomy: a prospective clinical trial. Nutrients 2016; 8: 357.

20. Meier M, Andersen KJ, Knudsen AR, et al. Liver regeneration is dependent on the extent of hepatectomy. J Surg Res 2016; 205: 76-84.

21. Andersen KJ, Knudsen AR, Kannerup AS, et al. The natural history of liver regeneration in rats: description of an animal model for liver regeneration studies. Int J Surg 2013; 11: 903-908.

22. Kwon AH, Uetsuji S, Yamamura M, et al. Effect of administration of fibronectin or aprotinin on liver regeneration after experimental hepatectomy. Ann Surg 1990; 211: 295-300.

23. Sánchez-Hidalgo JM, Naranjo A, Ciria R, et al. Impact of age on liver regeneration response to injury after partial hepatectomy in a rat model. J Surg Res 2012; 175: 1-9.

24. Holecek M. Three targets of branched-chain amino acid supplementation in the treatment of liver disease. Nutrition 2010; 26 482-490.

25. Kurokawa T, An J, Tsunekawa K, et al. Effect of L-arginine supplement on liver regeneration after partial hepatectomy in rats. World J Surg Oncol 2012; 10: 99.

26. Yao H, Fu X, Zi X, et al. Perioperative oral supplementation with fish oil promotes liver regeneration following partial hepatectomy in mice via AMPK activation. Mol Med Rep 2018; 17: 39053911.

27. Bémeur C, Butterworth RF. Nutrition in the management of cirrhosis and its neurological complications. J Clin Exp Hepatol 2014; 4: 141-150.

28. Mao SA, Glorioso JM, Nyberg SL. Liver regeneration. Transl Res 2014; 163: 352-362.

29. Qiu YD, Wang S, Yang Y, et al. Omega-3 polyunsaturated fatty acids promote liver regeneration after $90 \%$ hepatectomy in rats. World J Gastroenterol 2012; 18: 3288-3295.

30. Böhm F, Kohler UA, Speicher T, et al. Regulation of liver regeneration by growth factors and cytokines. EMBO Mol Med 2010; 2: 294-305.

31. Gazit V, Weymann A, Hartman E, et al. Liver regeneration is impaired in lipodystrophic fld mice. Hepatology 2010; 52: 21092117.

32. Rudnick DA, Huang J. Metabolic regulation of liver regeneration. In: Liver regeneration. Udayan A (ed.). Academic Press, Boston 2015; 213-228.

33. Zhu XH, Wu YF, Qiu YD, et al. Liver-protecting effects of omega-3 fish oil lipid emulsion in liver transplantation. World J Gastroenterol 2012; 18: 6141-6147.

34. Silva RM, Malafaia O, Torres OJ, et al. Evaluation of liver regeneration diet supplemented with omega- 3 fatty acids: experimental study in rats. Rev Col Bras Cir 2015; 42: 393-397.

35. Melo JU, Santos JM, Kimura Ode S, et al. Effects of fatty acids on liver regeneration in rats. Rev Col Bras Cir 2010; 37: 351-357. 